comments appreciated

\title{
A Simple Proof of the Necessity of the Transversality Condition
}

\author{
Takashi Kamihigashi \\ RIEB \\ Kobe University \\ Rokkodai, Nada, Kobe 657-8501 \\ Japan \\ Phone/Fax: +81-78-803-7015 \\ E-mail: tkamihig@rieb.kobe-u.ac.jp
}

January 16, 2001

\begin{abstract}
This note provides a simple proof of the necessity of the transversality condition for the differentiable reduced-form model. The proof uses only an elementary perturbation argument without relying on dynamic programming. The proof makes it clear that, contrary to common belief, the necessity of the transversality condition can be shown in a straightforward way.
\end{abstract}

Keywords: Transversality condition, reduced-form model, dynamic optimization. JEL Classification Numbers: C61, D90, G12 


\section{Introduction}

"The necessity of the transversality condition is a difficult issue," note Stokey and Lucas (1989, p. 102) after proving the sufficiency of the transversality condition. As a matter of fact, necessity of the transversality condition has long been widely perceived as a difficult issue, perhaps because the classical proofs of the necessity of the transversality condition are not easily understandable to nontechnical readers. What makes those proofs difficult, however, is not the difficulties in proving the transversality condition itself but the technical arguments required for proving the existence of support prices (Peleg, 1970; Peleg and Ryder, 1972; Weitzman, 1973; Araujo and Scheinkman, 1983) or for proving the envelope condition (Benveniste and Scheinkman, 1982). Though such arguments may be necessary when one wishes to establish a characterization theorem for a general maximization problem, they can in fact be entirely bypassed when one wishes only to prove the necessity of the transversality condition for the differentiable reduced-form model.

The purpose of this note is to offer a simple proof of the necessity of the transversality condition. The result proved in this note is a more or less well-known variant of Weitzman's (1973) theorem. The assumptions we use that are not assumed by Weitzman are the differentiability of the return functions and the interiority of a given optimal path. These assumptions allow us to work directly with derivatives, making it unnecessary to construct support prices. Another feature of our approach is that we do not use dynamic programming. Without relying on dynamic programming, we directly prove the necessity of the transversality condition using only an elementary perturbation argument.

While similar arguments are used in Kamihigashi (2000a, 2000b, 2000c), these papers do not provide a direct proof of the necessity of the transversality condition. Kamihigashi (2000a) focuses on Ekeland and Scheinkman's (1986) result. Instead of simplifying the proofs of well-known results, the other two papers seek to generalize well-known results and to establish new results. We believe that the direct proof offered in this note will benefit the profession by demystifying the necessity of the transversality condition.

The next section presents the model and states the result. Section 3 presents the proof. 
Section 4 comments on the proof. Section 5 concludes the note.

\section{The Transversality Condition}

Consider the following maximization problem.

$$
\left\{\begin{aligned}
\max _{\left\{x_{t}\right\}_{t=0}^{\infty}} & \sum_{t=0}^{\infty} v_{t}\left(x_{t}, x_{t+1}\right) \\
\text { s.t. } & x_{0}=\bar{x}_{0}, \quad \forall t \in \mathbb{Z}_{+},\left(x_{t}, x_{t+1}\right) \in X_{t} .
\end{aligned}\right.
$$

Since the assumptions and definitions used here are standard, they are stated without comment.

Assumption 2.1. $\exists n \in \mathbb{N}, \bar{x}_{0} \in \mathbb{R}_{+}^{n}$ and $\forall t \in \mathbb{Z}_{+}, X_{t} \subset \mathbb{R}_{+}^{n} \times \mathbb{R}_{+}^{n}$.

Assumption 2.2. $\forall t \in \mathbb{Z}_{+}, X_{t}$ is convex and $(0,0) \in X_{t}$.

Assumption 2.3. $\forall t \in \mathbb{Z}_{+}, v_{t}: X_{t} \rightarrow \mathbb{R}$ is $C^{1}$ on $\stackrel{\circ}{X_{t}}$ and concave.

For $t \in \mathbb{Z}_{+}$and $(y, z) \in \stackrel{\circ}{X}_{t}$, let $v_{t, 2}(y, z)$ denote the partial derivative of $v_{t}$ with respect to $z$; define $v_{t, 1}(y, z)$ similarly.

Assumption 2.4. $\forall t \in \mathbb{Z}_{+}, \forall(y, z) \in \stackrel{\circ}{X}_{t}, v_{t, 2}(y, z) \leq 0{ }^{1}$

We say that a path $\left\{x_{t}\right\}_{t=0}^{\infty}$ is feasible if $x_{0}=\bar{x}_{0}$ and $\forall t \in \mathbb{Z}_{+},\left(x_{t}, x_{t+1}\right) \in X_{t}$.

Assumption 2.5. For any feasible path $\left\{x_{t}\right\}$,

$$
\sum_{t=0}^{\infty} v_{t}\left(x_{t}, x_{t+1}\right) \equiv \lim _{T \uparrow \infty} \sum_{t=0}^{T} v_{t}\left(x_{t}, x_{t+1}\right)
$$

exists in $(-\infty, \infty)$.

We say that a feasible path $\left\{x_{t}^{*}\right\}$ is optimal if for any feasible path $\left\{x_{t}\right\}$,

$$
\sum_{t=0}^{\infty} v_{t}\left(x_{t}, x_{t+1}\right) \leq \sum_{t=0}^{\infty} v_{t}\left(x_{t}^{*}, x_{t+1}^{*}\right) .
$$

We say that a feasible path $\left\{x_{t}\right\}$ is interior if $\forall t \in \mathbb{Z}_{+},\left(x_{t}, x_{t+1}\right) \in \stackrel{\circ}{X_{t}}$. The following result is proved in Section 2.1.

\footnotetext{
${ }^{1}$ Due to the Euler equation (5), Theorem 2.1 below holds even if this inequality is replaced by $v_{t, 1}(y, z) \geq 0$.
} 
Theorem 2.1. Under Assumptions 2.1-2.5, for any interior optimal path $\left\{x_{t}^{*}\right\}$,

$$
\lim _{T \uparrow \infty}\left[-v_{T, 2}\left(x_{T}^{*}, x_{T+1}^{*}\right) x_{T+1}^{*}\right]=0 .
$$

This is a variant of Weitzman (1973, Theorem) and a discrete-time version of Benveniste and Scheinkman (1982, Theorem 3.A). Since an interior optimal path $\left\{x_{t}^{*}\right\}$ satisfies the Euler equation

$$
v_{t, 2}\left(x_{t}^{*}, x_{t+1}^{*}\right)+v_{t+1,1}\left(x_{t+1}^{*}, x_{t+2}^{*}\right)=0
$$

for $t \in \mathbb{Z}_{+}$, condition (4) can equivalently be expressed as

$$
\lim _{T \uparrow \infty} v_{T, 1}\left(x_{T}^{*}, x_{T+1}^{*}\right) x_{T}^{*}=0 .
$$

Condition (4), or the above equivalent form, is the most commonly used transversality condition.

As the proof below shows, however, condition (4) is a necessary condition regardless of validity of the Euler equation. In addition, condition (4) corresponds better to the continuous-time version of the transversality condition.

\section{Proof of Theorem 2.1}

We prepare the following elementary lemma.

Lemma 3.1. Let $f:[0,1] \rightarrow \mathbb{R}$ be a concave function. Then

$$
\forall \gamma \in[0,1), \forall \lambda \in[\gamma, 1), \quad \frac{f(1)-f(\lambda)}{1-\lambda} \leq \frac{f(1)-f(\gamma)}{1-\gamma}
$$

Proof. Let $\lambda \in[\gamma, 1)$ and $\mu=(1-\lambda) /(1-\gamma)$. By concavity, $f(\lambda) \geq \mu f(\gamma)+(1-\mu) f(1)=$ $-\mu(f(1)-f(\gamma))+f(1)$. Thus $f(1)-f(\lambda) \leq \mu(f(1)-f(\gamma))$; the inequality in (7) follows.

Now to prove Theorem 2.1, let $\left\{x_{t}^{*}\right\}$ be an interior optimal path. Let $T \in \mathbb{Z}_{+}$. By interiority and Assumption 2.2, for $\lambda \in[\underline{\lambda}, 1)$ sufficiently close to one, the path

$$
\left\{x_{0}^{*}, x_{1}^{*}, \cdots, x_{T}^{*}, \lambda x_{T+1}^{*}, \lambda x_{T+2}^{*}, \cdots\right\}
$$


is feasible. Let $\lambda \in[0,1)$ be so close to one that the above path is feasible. By optimality,

$$
v_{T}\left(x_{T}^{*}, \lambda x_{T+1}^{*}\right)-v_{T}\left(x_{T}^{*}, x_{T+1}^{*}\right)+\sum_{t=T+1}^{\infty}\left[v_{t}\left(\lambda x_{t}^{*}, \lambda x_{t+1}^{*}\right)-v_{t}\left(x_{t}^{*}, x_{t+1}^{*}\right)\right] \leq 0 .
$$

Dividing through by $(1-\lambda)$ yields

$$
\begin{aligned}
\frac{v_{T}\left(x_{T}^{*}, \lambda x_{T+1}^{*}\right)-v_{T}\left(x_{T}^{*}, x_{T+1}^{*}\right)}{1-\lambda} & \leq \sum_{t=T+1}^{\infty} \frac{v_{t}\left(x_{t}^{*}, x_{t+1}^{*}\right)-v_{t}\left(\lambda x_{t}^{*}, \lambda x_{t+1}^{*}\right)}{1-\lambda} \\
& \leq \sum_{t=T+1}^{\infty}\left[v_{t}\left(x_{t}^{*}, x_{t+1}^{*}\right)-v_{t}(0,0)\right],
\end{aligned}
$$

where the last inequality holds by Lemma 3.1 with $\gamma=0$. Applying $\lim _{\lambda \uparrow 1}$ to (10) and (11) yields

$$
0 \leq-v_{T, 2}\left(x_{T}^{*}, x_{T+1}^{*}\right) x_{T+1}^{*} \leq \sum_{t=T+1}^{\infty}\left[v_{t}\left(x_{t}^{*}, x_{t+1}^{*}\right)-v_{t}(0,0)\right]
$$

where the first inequality holds by Assumption 2.4. Applying $\lim _{T \uparrow \infty}$ to (12) yields

$$
0 \leq \lim _{T \uparrow \infty}\left[-v_{T, 2}\left(x_{T}^{*}, x_{T+1}^{*}\right) x_{T+1}^{*}\right] \leq \lim _{T \uparrow \infty} \sum_{t=T+1}^{\infty}\left[v_{t}\left(x_{t}^{*}, x_{t+1}^{*}\right)-v_{t}(0,0)\right]=0 .
$$

Condition (4) now follows.

\section{Comments}

The crucial step in the above proof is the inequality in (11). Very roughly speaking, in Ekeland and Scheinkman's (1986) proof, $\lim _{\lambda \uparrow 1}$ is directly applied to both sides of (10). Again very roughly speaking, in Benveniste and Scheinkman's (1982) proof, $\lim _{\lambda \uparrow 1}$ is applied to the following inequality.

$$
\frac{v_{T}\left(x_{T}^{*}, \lambda x_{T+1}^{*}\right)-v_{T}\left(x_{T}^{*}, x_{T+1}^{*}\right)}{1-\lambda} \leq \frac{V_{T+1}\left(x_{T+1}^{*}\right)-V_{T+1}\left(\lambda x_{T+1}^{*}\right)}{1-\lambda},
$$

where $V_{T+1}$ is the value function for the maximization problem starting from period $T+1 .^{2}$ Though both methods eventually work, they require nontrivial technical arguments since the right-hand sides of (10) and (14) depend on $\lambda$. In our proof, by contrast, the process of applying $\lim _{\lambda \uparrow 1}$ is trivial since the right-hand side of (11) does not involve $\lambda$.

\footnotetext{
${ }^{2}$ To be more specific, the corresponding Bellman equation is $V_{T}(x)=\max \left\{v_{T}(x, y)+V_{T+1}(y) \mid y:(x, y) \in\right.$ $\left.X_{T}\right\}$. By optimality, $v_{T}\left(x_{T}^{*}, \lambda x_{T+1}^{*}\right)+V_{T+1}\left(\lambda x_{T+1}^{*}\right) \leq v_{T}\left(x_{T}^{*}, x_{T+1}^{*}\right)+V_{T+1}\left(x_{T+1}^{*}\right)$. This implies (14).
} 
All the assumptions, Assumptions 2.1-2.5, can considerably be weakened. In fact, only the following three assumptions are needed for the basic argument of our proof to go through. First, for $T \in \mathbb{Z}_{+}$, the path specified by (8) is feasible for all $\lambda<1$ sufficiently close to one. Second, given $T \in \mathbb{Z}_{+}$, for all $\lambda<1$ sufficiently close to one, the right-hand side of (10) is bounded above by some sequence that does not depend on $\lambda$ and that converges to zero as $T \uparrow \infty$. Third, the left-hand side of (10) has a limit of some kind as $\lambda \uparrow 1 .^{3}$

The second assumption above is useful particularly when $v_{t}(0,0)=-\infty$, which is the case in many parametric models. In such cases, the above proof, which does not work in its current form, can easily be modified as follows. Assume

$$
\sum_{t=1}^{\infty}\left[v_{t}\left(x_{t}^{*}, x_{t+1}^{*}\right)-v_{t}\left(\gamma x_{t}^{*}, \gamma x_{t+1}^{*}\right)\right]
$$

exists in $(-\infty, \infty)$ for some $\gamma \in[0,1)$. By Lemma 3.1, for $\lambda \in[\gamma, 1)$,

$$
\sum_{t=T+1}^{\infty} \frac{v_{t}\left(x_{t}^{*}, x_{t+1}^{*}\right)-v_{t}\left(\lambda x_{t}^{*}, \lambda x_{t+1}^{*}\right)}{1-\lambda} \leq \sum_{t=T+1}^{\infty} \frac{v_{t}\left(x_{t}^{*}, x_{t+1}^{*}\right)-v_{t}\left(\gamma x_{t}^{*}, \gamma x_{t+1}^{*}\right)}{1-\gamma} .
$$

Use this inequality in place of (11). The rest of the proof then goes through.

The above argument shows that the transversality condition is necessary as long as expression (15) is finite for some $\gamma \in[0,1) .{ }^{4}$ This result is useful for models with unbounded return functions since it does not require the objective function to be finite, or even welldefined, for all feasible paths.

\section{Conclusion}

This note proved the necessity of the transversality condition for the differentiable reducedform model using only an elementary perturbation argument. The proof is short and simple because it bypasses the technical arguments required for constructing support prices and showing the envelope condition. We hope, and believe, that the direct proof offered in this note will help the profession better understand the transversality condition.

\footnotetext{
${ }^{3}$ See Kamihigashi (2000c) for general results established under minimal assumptions.

${ }^{4}$ Various results of this nature are established in Kamihigashi (2000b, 2000c).
} 


\section{References}

Araujo, A., and J.A. Scheinkman, 1983, "Maximum Principle and Transversality Condition for Concave Infinite Horizon Economic Models," Journal of Economic Theory 30, 1-16.

Benveniste, L.M., and J.A. Scheinkman, 1982, "Duality Theory for Dynamic Optimization Models of Economics: The Continuous Time Case," Journal of Economic Theory 27, $1-19$.

Ekeland, I., and J.A. Scheinkman, 1986, "Transversality Conditions for Some Infinite Horizon Discrete Time Optimization Problems," Mathematics of Operations Research 11, $216-229$.

Kamihigashi, T., 2000a, "A Simple Proof of Ekeland and Scheinkman's Result on the Necessity of a Transversality Condition," Economic Theory 15, 463-468.

Kamihigashi, T., 2000b, "Necessity of Transversality Conditions for Infinite Horizon Problems," Econometrica, forthcoming.

Kamihigashi, T., 2000c, "Necessity of Transversality Conditions for Stochastic Problems," RIEB Discussion Paper Series No. 115, Kobe University.

Peleg, B., 1970, "Efficiency Prices for Optimal Consumption Plans III," Journal of Mathematical Analysis and Applications 32, 630-638.

Peleg, B., and H.E. Ryder, Jr., 1972, "On Optimal Consumptions Plans in a Multi-sector Economy," Review of Economic Studies 39, 159-169.

Stokey, N., and R.E. Lucas, Jr., 1989, Recursive Methods in Economic Dynamics. Cambridge, MA: Harvard University Press.

Weitzman, M.L., 1973, "Duality Theory for Infinite Horizon Convex Models," Management Science 19, 783-789. 